Revista Brasil. Bot., V.25, n.1, p.55-64, mar. 2002

\title{
A invasão de um fragmento florestal em São Paulo (SP) pela palmeira australiana Archontophoenix cunninghamiana H. Wendl. \& Drude
}

\author{
RICARDO DISLICH ${ }^{1}$, NABOR KISSER ${ }^{1}$ e VÂNIA R. PIVELLO ${ }^{1,2}$
}

(recebido: 6 de setembro de 2000; aceito: 14 de novembro de 2001)

\begin{abstract}
The invasion of a forest fragment in São Paulo (SP) by the Australian palm Archontophoenix cunninghamiana H. Wendl. \& Drude). Biological invasions are a threat to the maintenance of biological diversity, especially in small, isolated habitat fragments. This work reports the invasion of the Reserve of the Cidade Universitária "Armando de Salles Oliveira" (233' S, 46 $43^{\prime}$ W), a small (10 ha) forest fragment, by a palm native of Australia, describing spatial patterns, size structure and dynamic features of its population. Adult individuals $(\mathrm{DBH}>25 \mathrm{~cm})$ occur in a large portion of the Reserve. In a $2.1 \mathrm{ha}$ area inside the Reserve, A. cunninghamiana was the species with the highest density among trees with DBH $\geq 9.5 \mathrm{~cm}$, with 305 individuals (22.5\% of total). The species was absent from sites with a recent history of human disturbance, suggesting preference for establishment in shady sites. The size structure analysis indicates a future increase in the relative density of the species. Seedlings (with pinatissect leaves, without woody stem) were absent from only three out of 87 studied quadrats (10 m x $10 \mathrm{~m}$ ) and juveniles (with woody stem) with $\mathrm{DBH}<9.5 \mathrm{~cm}$ were absent from 19 quadrats. Two surveys with a 2.5 years interval (DBH $\geq 9.5$ $\mathrm{cm}$ ) showed the death of three of the initial 154 individuals and the recruitment of 89 more, leading to a population growth of 19.4\% year $^{-1}$, very high in comparison with literature data. The high density already achieved by the invader and its tendency to increase even more call for management actions in order to reverse the invasion process.
\end{abstract}

RESUMO - (A invasão de um fragmento florestal em São Paulo (SP) pela palmeira australiana Archontophoenix cunninghamiana H. Wendl. \& Drude). Invasões biológicas são uma ameaça à manutenção da diversidade biológica, especialmente em fragmentos de hábitat pequenos e isolados. Este trabalho reporta a invasão da Reserva Florestal da Cidade Universitária "Armando de Salles Oliveira" (2334' S e 4643' W), um pequeno (10 ha) fragmento florestal, por uma palmeira nativa da Austrália, através da descrição dos padrões espaciais, estrutura de tamanhos e aspectos dinâmicos de sua população. Indivíduos adultos (DAP > 25 $\mathrm{cm}$ ) se distribuem por grande parte da Reserva. Entre as árvores com DAP $\geq 9,5 \mathrm{~cm}$, em uma área de 2,1 ha, A. cunninghamiana foi a espécie com maior densidade, com 305 indivíduos (22,5\% do total). A espécie esteve ausente em locais com histórico recente de perturbação antrópica, sugerindo preferência por estabelecimento em locais sombreados. A análise da estrutura de tamanhos indica um aumento futuro da densidade relativa da espécie. Plântulas (com folhas pinatissectas, sem caule lignificado) não ocorreram em apenas três de 87 parcelas de $10 \mathrm{~m}$ x $10 \mathrm{~m}$ estudadas, e jovens (com caule lignificado) com DAP $<9,5 \mathrm{~cm}$ não ocorreram em 19 delas. Dois levantamentos com 2,5 anos de intervalo (DAP $\geq 9,5 \mathrm{~cm}$ ) mostraram a morte de três dos 154 indivíduos iniciais e o recrutamento de mais 89 , levando a um crescimento populacional de $19,4 \%$.ano ${ }^{-1}$, muito elevado se comparado com dados da literatura. A grande densidade já alcançada pela invasora, assim como a tendência de aumento dessa densidade, pedem por ações de manejo que visem a reversão deste processo de invasão.

Key words - Archontophoenix cunninghamiana, biological invasion, secondary forest

\section{Introdução}

As invasões biológicas constituem um fenômeno ainda pouco estudado, porém reconhecido na década de 90 como uma das maiores ameaças à biodiversidade do planeta (Cronk \& Fuller 1995, Pysek et al. 1995, Williamson 1996, Meffe \& Carroll 1997). Segundo definido por Pysek (1995), espécie invasora é "uma exótica cuja distribuição e/ou abundância está em processo de aumento", e espécie exótica é "aquela que

\footnotetext{
1. Universidade de São Paulo, Instituto de Biociências, Departamento de Ecologia, Rua do Matão, Travessa 14, n. 101, 05508-900 São Paulo, SP.

2. Autor para correspondência: vrpivel@ib.usp.br
}

alcançou a área como conseqüência de atividades do homem neolítico ou pós-neolítico ou seus animais domésticos". As invasões biológicas podem causar impactos em diversos níveis, incluindo efeitos sobre indivíduos (morfologia, comportamento, mortalidade, crescimento), efeitos genéticos (alteração de padrões de fluxo gênico, hibridização), efeitos sobre a dinâmica de populações (abundância, crescimento populacional, extinção), a comunidade (riqueza de espécies, diversidade, estrutura trófica) e processos do ecossistema (disponibilidade de nutrientes, produtividade, regime de perturbações) (Parker et al. 1999).

As características que aumentam as chances de uma espécie se tornar invasora e as características que tornam determinado ecossistema mais suscetível a 
invasões biológicas são temas que vêm sendo investigados (Noble 1989, Roy 1990, Lodge 1993, Rejmanek \& Richardson 1996, Williamson \& Fitter 1996a). Existem plantas invasoras de todas as principais formas de vida, de ervas a árvores, ocorrendo nos mais diversos tipos de ecossistemas (Cronk \& Fuller 1995). Florestas tropicais pouco perturbadas parecem ser raramente invadidas (Laurance \& Bierregaard 1997), embora haja exceções a esta regra (Cronk \& Fuller 1995, Peters 2001). Ainda em florestas tropicais pouco perturbadas, lianas e trepadeiras exóticas podem ter efeito importante nas bordas, sendo também freqüente a ocorrência de plantas exóticas colonizando clareiras. Estas, porém, geralmente têm existência efêmera e vão progressivamente se retirando da comunidade, com o fechamento do dossel (Laurance \& Bierregaard 1997). Hábitats florestais perturbados parecem ser muito mais suscetíveis às invasões biológicas. Muitas invasoras são heliófitas, encontrando mais oportunidades de estabelecimento nestes locais perturbados. Em geral, fragmentos pequenos de hábitats naturais são mais suscetíveis a invasão biológica do que grandes áreas contínuas (Cronk \& Fuller 1995, Laurance \& Bierregaard 1997).

Para a América do Sul, ainda existem poucos registros de plantas invasoras em ambientes florestais (Cronk \& Fuller 1995), provavelmente em decorrência da escassez de estudos com este enfoque, embora processos de invasão biológica em ambientes abertos de savana tenham sendo reportados recentemente (Pivello et al. 1999a, b).

Embora ainda não exista um consenso para uma abordagem de quantificação do impacto de invasões biológicas, Parker et al. (1999) sugerem que três fatores devem ser levados em conta para medi-lo: área total ocupada, abundância local, e alguma medida do impacto por indivíduo. Quanto maior qualquer um desses fatores, maior o impacto causado pela espécie. O raciocínio utilizado no uso da abundância do invasor como medida do impacto é de que qualquer biomassa (ou espaço, ou energia) controlada pelo invasor representa recursos não mais disponíveis para competidores (Parker et al. 1999).

Este trabalho trata da invasão de um pequeno fragmento de floresta em área urbana, na cidade de São Paulo, por Archontophoenix cunninghamiana $\mathrm{H}$. Wendl. \& Drude (Arecaceae), uma espécie exótica. Para caracterizar a existência do processo de invasão, foi descrita a dinâmica (recrutamento, mortalidade, crescimento, incremento diamétrico) da espécie na área. Como indicação do impacto causado, foram analisadas a área ocupada e abundância da espécie, como sugerido por Parker et al. (1999). Para tanto, foram descritos seus padrões espaciais e estrutura de tamanhos no interior do fragmento. A fim de avaliar a abundância relativa de A. cunninghamiana na Reserva, foi realizado também levantamento dos indivíduos de outras espécies.

\section{Material e métodos}

Espécie estudada - Archontophoenix cunninghamiana é nativa de florestas tropicais da Austrália oriental, entre as latitudes $35,5^{\circ} \mathrm{S}$ e $21^{\circ} \mathrm{S}$ (Waterhouse \& Quinn 1978). Tem o hábito de uma palmeira monocaule que floresce por vários anos, através de uma sucessão de inflorescências "laterais" (Waterhouse \& Quinn 1978). Seu estipe apresenta crescimento em diâmetro com o tempo, pertencendo ao "tipo B" descrito por Schoute (1912). Em seu hábitat natural, indivíduos dessa espécie podem alcançar altura superior a $20 \mathrm{~m}$ e diâmetro à altura do peito superior a $20 \mathrm{~cm}$ (Waterhouse \& Quinn 1978). A. cunninghamiana é amplamente utilizada como ornamental (Pirani \& Cortopassi-Laurino 1994) em praças, jardins e na arborização urbana em São Paulo, onde floresce durante o ano inteiro, sendo visitada por várias espécies de abelhas sociais à procura de néctar e pólen (Pirani \& CortopassiLaurino 1994). Suas drupas vermelhas, com 1-2 cm de diâmetro (Pirani \& Cortopassi-Laurino 1994), disponíveis ao longo do ano inteiro, são consumidas por um número relativamente grande de espécies de aves urbanas (Hasui \& Höfling 1998). Local de estudo - A Cidade Universitária “Armando de Salles Oliveira” (CUASO), da Universidade de São Paulo (USP), localiza-se no bairro do Butantã, em São Paulo, SP. Em seu interior localiza-se a Reserva estudada neste trabalho

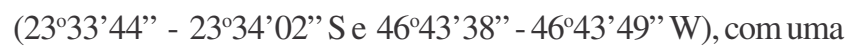
área de $102.100 \mathrm{~m}^{2}$, situada no vale de um riacho que se estende na direção S-N, em terreno com altitudes entre $775 \mathrm{~m}$ e $735 \mathrm{~m}$. Na parte mais baixa do terreno o represamento do riacho dá origem a um pequeno lago.

A área situa-se na Bacia de São Paulo, formada por depósitos do Terciário e Quaternário (Joly 1950). O solo é essencialmente argiloso, ácido, pobre em nutrientes e com altos teores de alumínio (Varanda 1977). O clima apresenta média anual de temperatura de $19,2{ }^{\circ} \mathrm{C}$ e precipitação média anual de $1207 \mathrm{~mm}$. As temperaturas médias mensais oscilam entre $14{ }^{\circ} \mathrm{C}$ (junho) e $23{ }^{\circ} \mathrm{C}$ (fevereiro). As precipitações médias mensais variam de $230 \mathrm{~mm}$ em janeiro a $40 \mathrm{~mm}$ em agosto, quando pode ocorrer déficit hídrico no solo (Gorresio-Roizman 1993).

Grande parte da área da Reserva é coberta por mata secundária (Cersósimo 1993), considerada por Rossi (1994) como um mosaico composto por áreas em diversos estádios de degradação e regeneração, efeito de seu histórico de perturbações antrópicas. A Reserva, cercada em 1979 para sua maior proteção, representa um dos poucos remanescentes da cobertura florestal na cidade de São Paulo, com cerca de 120 espécies arbustivo-arbóreas nativas (Rossi 1994). Situada no domínio das Florestas Ombrófilas Densas, a mata apresenta 
relações florísticas com a Floresta Ombrófila Densa e a Floresta Estacional Semidecidual (sensu Veloso et al. 1991) do estado de São Paulo.

Padrões espaciais e abundância - O levantamento de indivíduos de Archontophoenix cunninghamiana e de outras espécies foi realizado em três escalas espaciais, representadas por três áreas contínuas $(1,2$ e 3 ) de tamanho diferente. Em cada uma foi utilizado um critério de inclusão diferente. A alocação das áreas de levantamento, descritas a seguir, buscou sempre a inclusão do gradiente borda-interior (riacho).

a) Área 1 - constitui a Reserva como um todo, excetuando-se o lago e uma pequena porção ao norte, com presença exclusiva de Eucalyptus sp. (figura 1). Nesta área, de 8,54 ha, foram identificados em nível de espécie e mapeados todos os indivíduos arbóreos com caule de diâmetro à altura do peito (DAP, medido a 1,30 m de altura) superior a $25 \mathrm{~cm}$. Este levantamento foi realizado entre junho de 1997 e novembro de 1999.

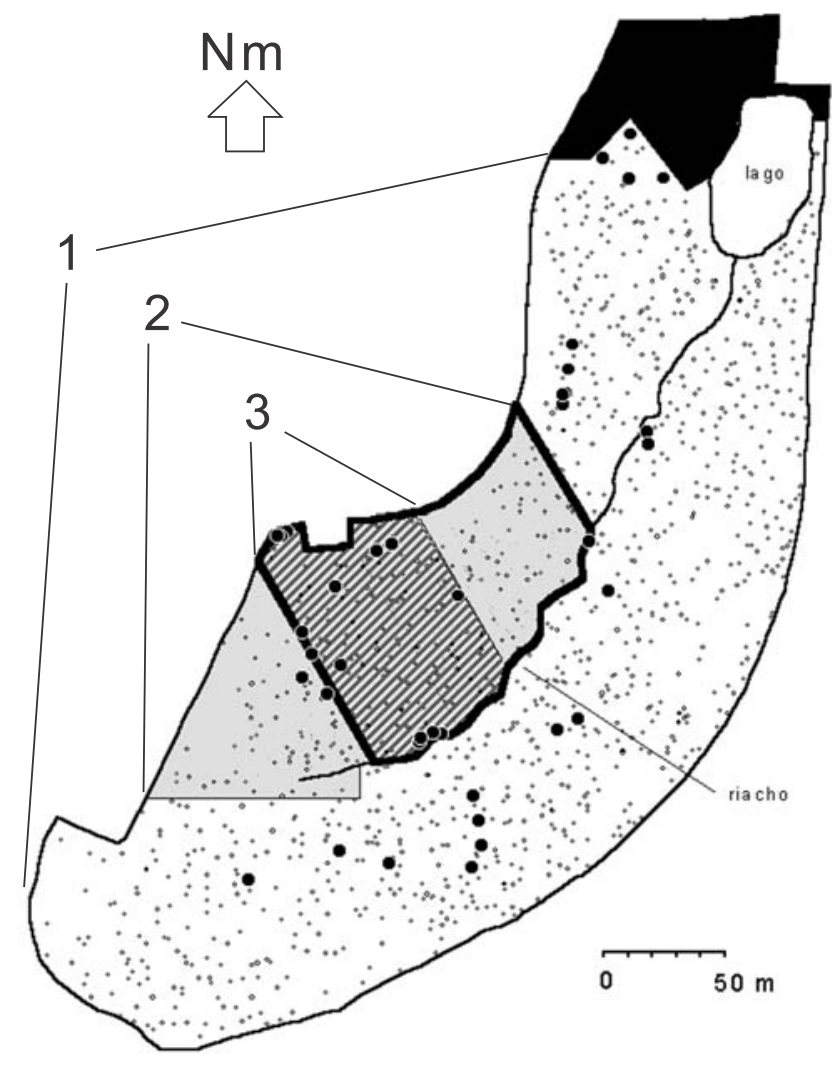

Figura 1. Áreas estudadas no interior da Reserva Florestal da Cidade Universitária Armando de Salles Oliveira (São Paulo, SP) e padrão espacial dos indivíduos com DAP > $25 \mathrm{~cm}$ de Archontophoenix cunninghamiana (círculos pretos) e de outras espécies (pontos pequenos). 1 = Área 1;2 = Área 2; 3 = Área 3. A linha mais grossa delimita a área de estudo da dinâmica. A área em negro (eucaliptal) não foi abrangida por este estudo. A linha sinuosa representa o riacho que corta a Reserva, terminando em um lago. Nm: norte magnético. b) Área 2 - constitui uma área contínua de 2,1 ha, inserida na Área 1, e se estende do limite da Reserva até o riacho, no lado oeste da Reserva (figura 1). Aqui, todas as árvores com DAP $\geq 9,5 \mathrm{~cm}$ foram mapeadas, identificadas e tiveram seu DAP medido. Este levantamento foi realizado entre junho de 1997 e março de 2000.

c) Área 3 - constitui uma área contínua com cerca de 0,86 ha, no interior da Área 2, estendendo-se também do limite da Reserva até o riacho (figura 1). Esta área foi dividida em parcelas de $10 \mathrm{~m}$ x $10 \mathrm{~m}$ e 87 delas foram analisadas, deixando-se de fora uma pequena área próxima à borda que sofre corte anual da vegetação. Na Área 3 foram registrados somente os indivíduos de A. cunninghamiana, em três classes de desenvolvimento: plântulas, jovens e adultos. As plântulas, aqui definidas como indivíduos com folhas já completamente pinatissectas mas ainda sem caule lignificado, foram contadas em cada uma das parcelas e nenhum tipo de medida foi realizado nestes indivíduos. Os jovens, indivíduos com caule lignificado, mas que ainda não atingiram o estado reprodutivo, e os adultos, indivíduos reprodutivos reconhecidos pela presença de cachos de flores ou frutos, foram contados em cada parcela. Para análise da estrutura de tamanhos de indivíduos pequenos, foi necessária a utilização de outra medida de tamanho diferente do DAP, uma vez que indivíduos com altura $<1,80 \mathrm{~m}$ usualmente não apresentam caule na altura de 1,30 m. Assim, foram tomadas as seguintes medidas para jovens e adultos: altura total da árvore, diâmetro na base do caule (DB - o maior diâmetro do caule entre $5 \mathrm{~cm}$ e $15 \mathrm{~cm}$ de altura) e DAP, quando possível. O levantamento da Área 3 foi realizado entre novembro de 1998 e junho de 1999.

Dinâmica - Para uma avaliação da dinâmica da população de Archontophoenix cunninghamiana dentro da Reserva (mortalidade, recrutamento, crescimento da população, incremento diamétrico), foram mapeados e medido o DAP de todos os indivíduos com DAP $\geq 9,5 \mathrm{~cm}$, em uma área contínua de 1,45 ha, parte da Área 2 (figura 1), entre junho de 1997 e fevereiro de 1998; os procedimentos foram repetidos em março de 2000 (intervalo médio de 2,5 anos). Mortalidade, recrutamento e crescimento da população foram calculados segundo o modelo exponencial proposto por Korning \& Balslev (1994):

$$
\mathrm{r}=\left(\mathrm{C}_{\mathrm{t}} / \mathrm{C}_{0}\right)^{1 / \mathrm{t}}-1
$$

sendo " $r$ " a taxa anual média de mortalidade $(r<0)$, a taxa anual média de recrutamento $(\mathrm{r}>0)$ ou a taxa anual média de crescimento da população, " $\mathrm{t}$ " o tempo em anos, " $\mathrm{C}_{0}$ " $\mathrm{e}$ " $\mathrm{C}_{\mathrm{t}}$ " o número de indivíduos no tempo 0 e após " $\mathrm{t}$ " anos, respectivamente.

O incremento diamétrico individual foi definido como a diferença de DAP, em cm, por unidade de tempo, em anos. Investigou-se a relação entre o incremento diamétrico e o DAP inicial através de ajuste por regressão de três tipos de funções: linear, logarítmica e polinomial (de segunda e terceira ordem), em busca do melhor ajuste possível. Foi considerada como de melhor ajuste a função com maior coeficiente de determinação $\mathrm{R}^{2}$. Essa função foi utilizada na construção de um modelo de 
incremento diamétrico ao longo do tempo para o "indivíduo médio" - assumindo distribuição normal do incremento de DAP - de Archontophoenix cunninghamiana: começando com DAP de 9,5 cm, o menor DAP para o qual a função ajustada é válida, calculou-se o incremento estimado para o próximo período de um ano, somando este valor ao DAP inicial para obter o DAP no próximo ano. Reiterando o processo, simulouse o incremento diamétrico ao longo do tempo (procedimento modificado de Terborgh et al. 1997).

\section{Resultados}

Padrões espaciais e abundância - Os indivíduos de Archontophoenix cunninghamiana com DAP $>25$ $\mathrm{cm}$ encontraram-se distribuídos por grande parte da Reserva, ocupando inclusive as áreas mais centrais, próximas ao riacho (Área 1, figura 1). Não ocorreram, porém, no extremo sul e na faixa mais a leste da Reserva, áreas que foram submetidas a fortes perturbações antrópicas nas últimas décadas, como aterros e corte da vegetação, inclusive com remoção completa do componente arbóreo. A. cunninghamiana apresentou 38 indivíduos com DAP > $25 \mathrm{~cm}$, ou 3,3\% do total de indivíduos na comunidade. Foi, assim, a sétima espécie em número de indivíduos dentre as 92 espécies encontradas na Área 1 (DAP > $25 \mathrm{~cm})$, atrás de Alchornea sidifolia Müll. Arg. (205 ind., 17,7\%), Piptadenia gonoacantha (Mart) J.F. Macbr. (145 ind., 12,5\%), Croton floribundus Spreng. (143 ind., 12,4\%), Cordia ecalyculata Vell. (55 ind., 4,8\%), Eucalyptus sp. (plantada como ornamental na Reserva) (41 ind., 3,5\%) e Cecropia glazioui Snethl. (40 ind., 3,5\%).

$\mathrm{Na}$ Área 2, observou-se maior densidade de indivíduos de Archontophoenix cunninghamiana (DAP $\geq 9,5 \mathrm{~cm}$ ) nas porções mais próximas à borda norte da Reserva (figura 2). Ao longo da borda reta a oeste, porém, há uma faixa de 20 a $30 \mathrm{~m}$ totalmente livre de A. cunninghamiana. Esta mesma faixa apresentou uma densidade relativamente pequena

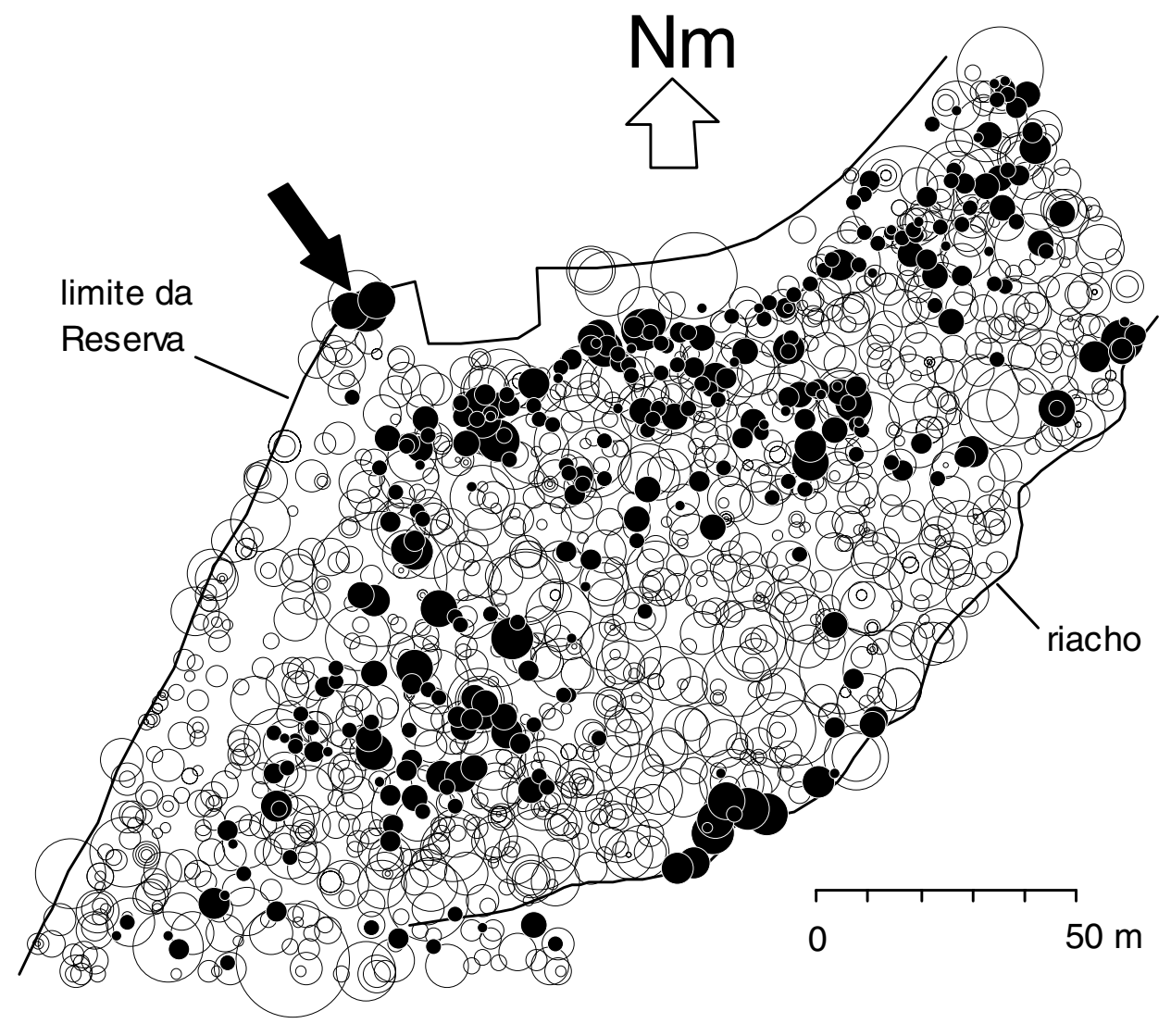

Figura 2. Padrão espacial dos indivíduos com DAP $\geq 9,5 \mathrm{~cm}$ de Archontophoenix cunninghamiana (círculos pretos) e de outras espécies (círculos vazados) na Área 2 (Reserva Florestal da CUASO, São Paulo, SP). O diâmetro dos círculos é proporcional ao DAP das árvores correspondentes. A seta preta indica os três indivíduos de A. cunninghamiana que provavelmente foram plantados como ornamentais. Nm: norte magnético. 
também de árvores de outras espécies (figura 2), e também sofreu intensas perturbações antrópicas nas últimas décadas, com corte da vegetação. Três indivíduos grandes de A. cunninghamiana na extremidade norte desta faixa foram provavelmente plantados como ornamentais, antes da Reserva ser cercada. Isto é indicado pelo fato de que todas as árvores maiores nas suas proximidades são de espécies exóticas cultivadas como ornamentais. Existe também uma aglomeração de indivíduos de A. cunninghamiana ao longo do riacho, inclusive com várias árvores adultas (figura 2). Com 305 (DAP $\geq 9,5 \mathrm{~cm}$ ) indivíduos, a espécie representou aqui $22,5 \%$ do total de indivíduos da comunidade, sendo a espécie de maior densidade na área, com cerca de 2,5 mais indivíduos que Cordia ecalyculata (124 ind., 9,16\%), três vezes mais que Alchornea sidifolia (107 ind.,7,91\%) e 4,5 vezes mais que Croton floribundus (70 ind., 5,17\%), as outras três espécies mais abundantes na área (DAP $\geq 9,5 \mathrm{~cm})$. A densidade relativa de $A$. cunninghamiana diminui com o critério de inclusão adotado (figura 3). Entre os indivíduos com DAP > 18 $\mathrm{cm}$ não é mais a espécie de maior abundância (figura 3). Esta diminuição da densidade relativa com o aumento do critério de inclusão pode ser explicada pela própria distribuição de freqüências de DAP da espécie, em relação à do restante da comunidade (figura 4A). $\mathrm{O}$ maior DAP encontrado em um indivíduo de $A$. cunninghamiana dentro da Reserva foi de $32,5 \mathrm{~cm}$, muito abaixo dos diâmetros alcançados por outras

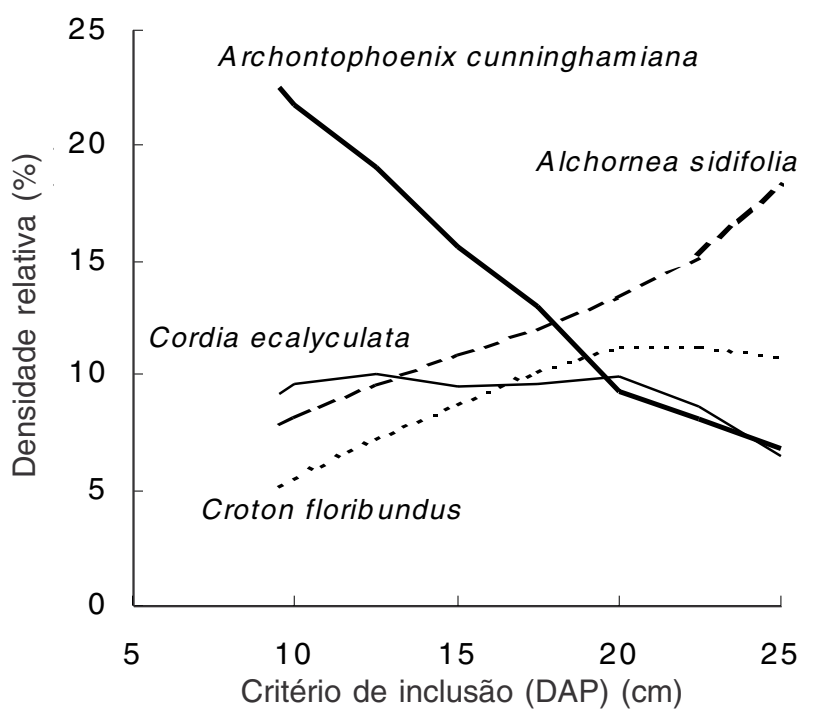

Figura 3. Densidades relativas (número de indivíduos da espécie/número total de indivíduos) de algumas das espécies mais abundantes na Área 2, em função do critério de inclusão adotado. árvores na área (figura 4B). A distribuição de freqüências de DAP de A. cunninghamiana, assim como a do restante da comunidade, se ajustaram bem a um modelo exponencial negativo, sendo que $A$. cunninghamiana apresentou uma distribuição com mais indivíduos menores em relação ao número de indivíduos maiores que o restante da comunidade (regressão linear do logaritmo natural do número de indivíduos em função da faixa de DAP: A. cunninghamiana: $\ln \mathrm{N}=$ $-0,1574$.DAP $+6,2948, \mathrm{R}^{2}=0,9697$; restante da comunidade: $\ln \mathrm{N}=-0,0973$. DAP $+6,3918, \mathrm{R}^{2}=$ 0,9398).
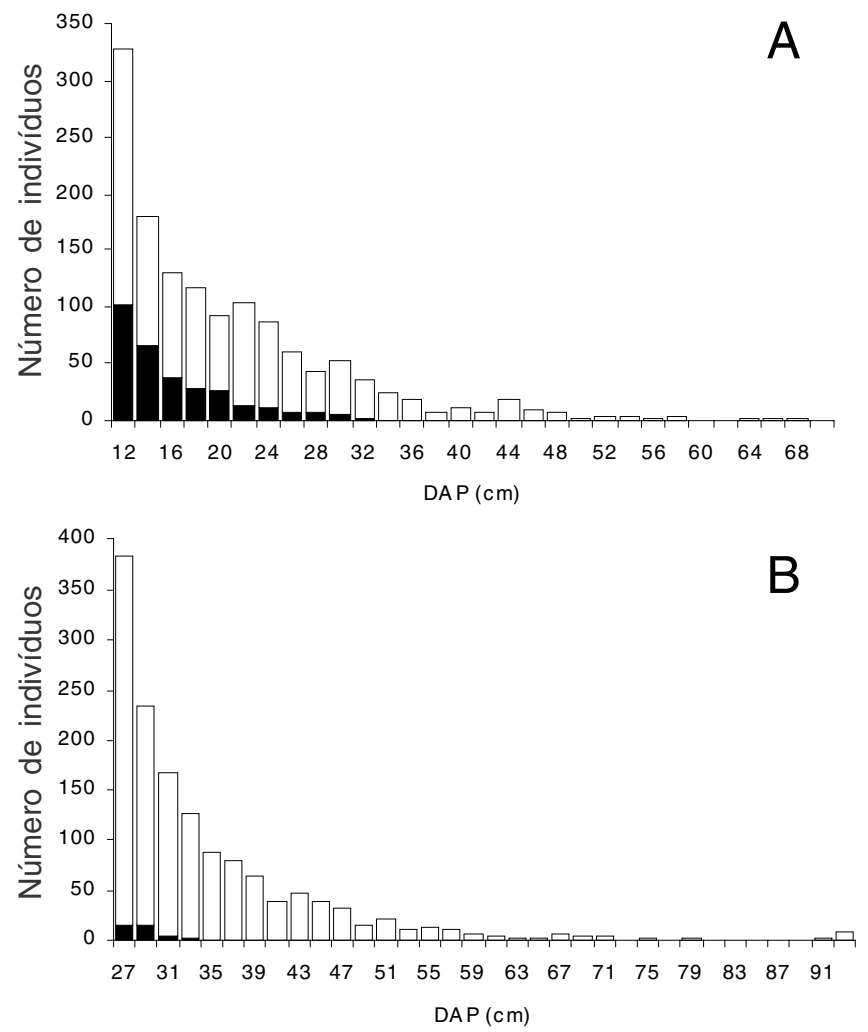

Figura 4. Distribuição de freqüências de DAP de Archontophoenix cunninghamiana (em preto) como parte da comunidade geral: (A) na Área 2, DAP $\geq 9,5 \mathrm{~cm}$; (B) na Área 1, DAP > $25 \mathrm{~cm}$. (Números no eixo das abscissas indicam o limite superior da classe correspondente).

Na Área 3, as 750 plântulas registradas, assim como os 265 indivíduos jovens com DAP $<9,5 \mathrm{~cm}$, encontraram-se mais concentrados nas regiões mais próximas à borda da Reserva (figura 5), embora também ocorressem, em números menores, em praticamente toda a extensão da área estudada. Somente em três das 87 parcelas $(3,4 \%)$ não foram encontradas plântulas de Archontophoenix cunninghamiana. Indivíduos jovens com DAP $<9,5 \mathrm{~cm}$ estiveram ausentes em 19 das 87 

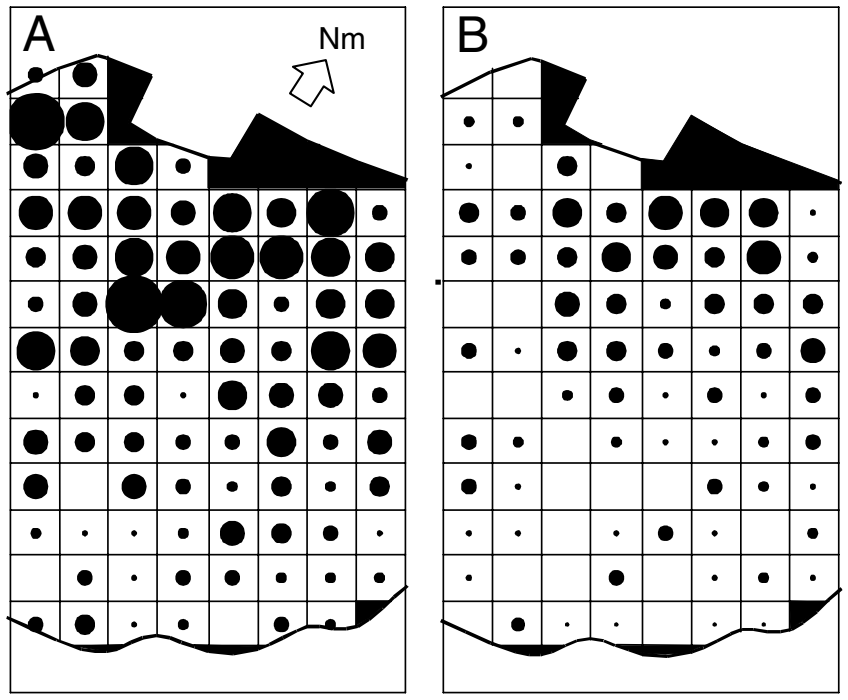

Figura 5. Número de plântulas (A) e de jovens com DAP < 9,5 cm (B) de Archontophoenix cunninghamiana nas parcelas $(10 \mathrm{~m} \times 10 \mathrm{~m})$ da Área 3. A área dos círculos é proporcional ao número de indivíduos em cada parcela. Os números máximos de indivíduos em uma parcela são de 46 plântulas e 16 jovens. As parcelas em negro não foram analisadas. Nm: norte magnético.

parcelas $(21,8 \%)$. Entre os indivíduos jovens e adultos na Área 3, DAP, DB e altura máxima se mostraram fortemente correlacionados (coeficiente de correlação não-paramétrico de Spearman $\left(\mathrm{r}_{\mathrm{s}}\right)$ : DAP x DB: $\mathrm{r}_{\mathrm{S}}=$ 0,972; DAP x altura: $\mathrm{r}_{\mathrm{S}}=0,879$; DB x altura: $\mathrm{r}_{\mathrm{S}}=0,937$; $\mathrm{p}<0,0001$ nos três casos). As distribuições de frequiências de altura (figura 6) e de DB na Área 3 também se ajustaram bem a um modelo exponencial negativo $\left(R^{2}=0,9741\right.$ e $R^{2}=0,9822$, respectivamente).

Todos os indivíduos com DAP $>25 \mathrm{~cm}$ analisados na Área 3 eram reprodutivos (adultos), enquanto todos com DAP menor ou igual a $25 \mathrm{~cm}$ eram jovens ou plântulas. Conseqüentemente, o critério de inclusão determinado para a Área 1 corresponde ao limite de DAP entre jovens e adultos. Assim, podemos considerar que na Área 1 (DAP > $25 \mathrm{~cm}$ ) foram analisados os indivíduos adultos de Archontophoenix cunninghamiana.

Dinâmica - No primeiro levantamento, em 1997, foram encontrados 154 indivíduos com DAP $\geq 9,5 \mathrm{~cm}$ na área estudada (figura 7). Depois de um período médio de 2,5 anos, três desses indivíduos haviam morrido e 89 novos indivíduos haviam sido recrutados. Isto implica em uma mortalidade de $0,78 \%$. ano $^{-1}$ e um recrutamento de $20,01 \%$. ano $^{-1}$, resultando em um crescimento líquido da população de $19,42 \%$.ano-1 ${ }^{-1}$ Os três indivíduos mortos foram encontrados muito próximo à borda e os

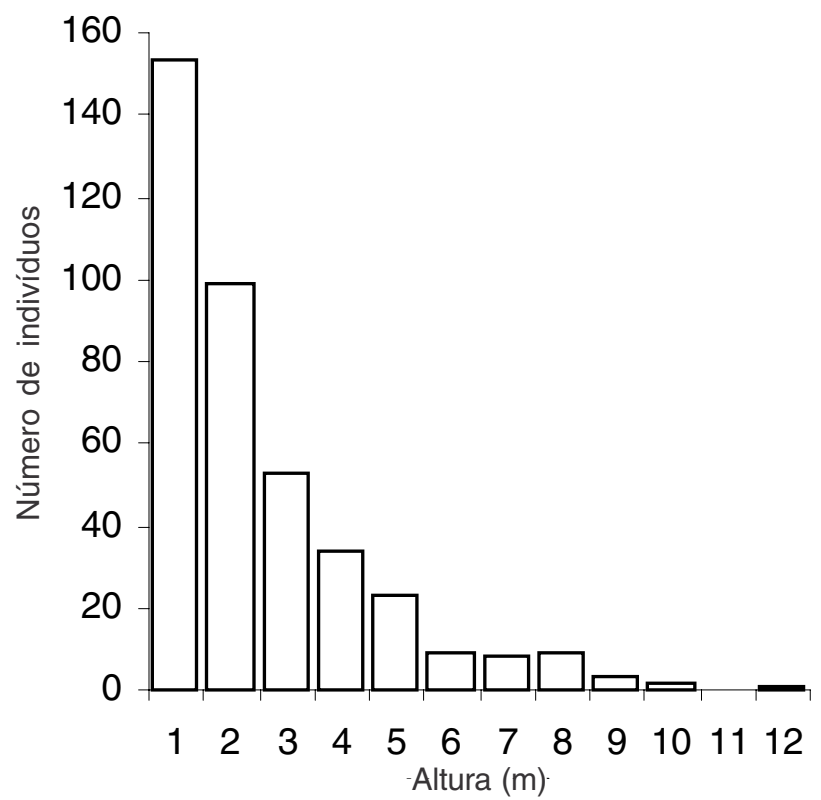

Figura 6. Distribuição de freqüências de altura dos indivíduos (exceto plântulas) de Archontophoenix cunninghamiana na Área 3. (Números no eixo das abscissas indicam o limite superior da classe correspondente).

recrutados ocorreram em maior densidade nas áreas próximas à borda da Reserva, onde já havia uma maior concentração de indivíduos de A. cunninghamiana (figura 7). Vários indivíduos recrutados no período,

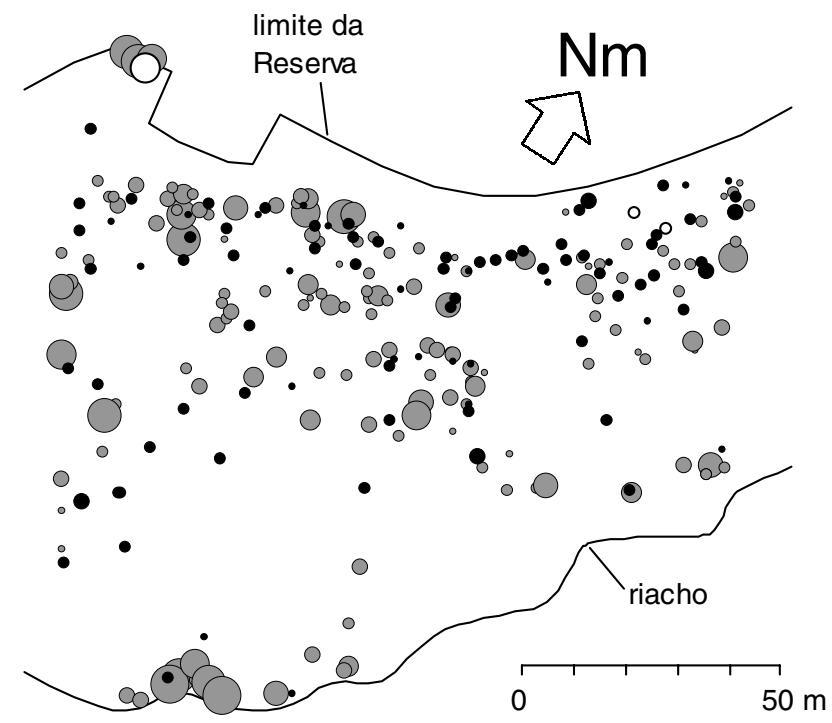

Figura 7. Padrão espacial dos indivíduos de Archontophoenix cunninghamiana com DAP $\geq 9,5 \mathrm{~cm}$ recrutados (círculos pretos), sobreviventes (círculos em cinza) e mortos (círculos vazados) em período de 2,5 anos. O diâmetro dos círculos é proporcional ao DAP das árvores correspondentes. Nm: norte magnético. 
porém, apareceram em áreas antes não ocupadas pela espécie, restringindo ainda mais as reduzidas regiões em que A. cunninghamiana não se fazia presente na área analisada (figura 7).

A taxa de incremento de DAP foi dependente do DAP inicial do indivíduo (coeficiente de correlação não-paramétrico de Spearman $=-0,37, \mathrm{n}=151, \mathrm{p}<$ 0,00001) (figura 8A). Em média, indivíduos de Archontophoenix cunninghamiana tenderam a ter um incremento alto - entre 1,0 e 1,5 cm.ano ${ }^{-1}$ - quando ainda relativamente pequenos, com DAP entre aproximadamente 10 e $18 \mathrm{~cm}$. A partir daí o incremento decresce fortemente com o aumento do DAP do indivíduo, alcançando crescimento nulo com DAP de $27 \mathrm{~cm}$, em média. A função que melhor descreveu este comportamento foi uma polinomial de terceiro grau; o $\mathrm{R}^{2}$ obtido, porém, foi baixo, indicando uma grande dispersão dos dados ao redor da curva média (figura 8A). Em outras palavras, houve grande variação de incremento diamétrico entre os indivíduos, mesmo levando-se em conta seu DAP inicial.

O modelo de incremento diamétrico ao longo do tempo para Archontophoenix cunninghamiana (figura 8B) mostra que o indivíduo médio, a partir dos $10 \mathrm{~cm}$ de DAP, precisaria entre 8 e 9 anos para alcançar $20 \mathrm{~cm}$ de DAP, e pouco mais de 18 anos para alcançar $25 \mathrm{~cm}$ de DAP. A partir daí o incremento diamétrico se daria de forma muito lenta, levando cerca de 6 anos para passar de 25 para $26 \mathrm{~cm}$ de DAP. Segundo o modelo, $83,9 \%$ dos indivíduos na Área 2 (DAP $\geq 9,5 \mathrm{~cm}$ ) têm até oito anos a mais do que quando tinham $9,5 \mathrm{~cm}$ de DAP, e 4,6\% dos indivíduos têm mais de 25.

\section{Discussão}

O presente artigo apresenta um exemplo dos riscos para a conservação biológica de pequenos fragmentos florestais no sudeste brasileiro, mesmo que adequadamente protegidos de perturbação antrópica mais direta. No caso específico, trata-se da invasão por Archontophoenix cunninghamiana. O presente estudo também possibilita o monitoramento futuro da dinâmica populacional da espécie. Acreditamos, porém, que os dados obtidos até o momento já justificam a adoção de medidas de controle da população, na tentativa de evitar danos maiores no futuro (Ruesink et al. 1995). Embora as conclusões derivadas de dados obtidos nas Áreas 2 e 3 não sejam extrapoláveis para a Reserva como um todo, já que não são resultado de uma amostragem aleatória, a ocorrência de invasão biológica em parte da Reserva já é suficientemente preocupante.
A invasão por Archontophoenix cunninghamiana na Reserva Florestal daCUASOé claramente demonstrada pela sua alta taxa de crescimento populacional. Taxas de crescimento populacional da magnitude da apresentada por A. cunninghamiana neste estudo $\left(19,4 \%\right.$.ano ${ }^{-1}$, DAP $\left.\geq 9,5 \mathrm{~cm}\right)$, devida principalmente a sua alta taxa de recrutamento $\left(19,7 \%\right.$.ano $\left.{ }^{-1}\right)$, são raramente reportadas para espécies arbóreas em
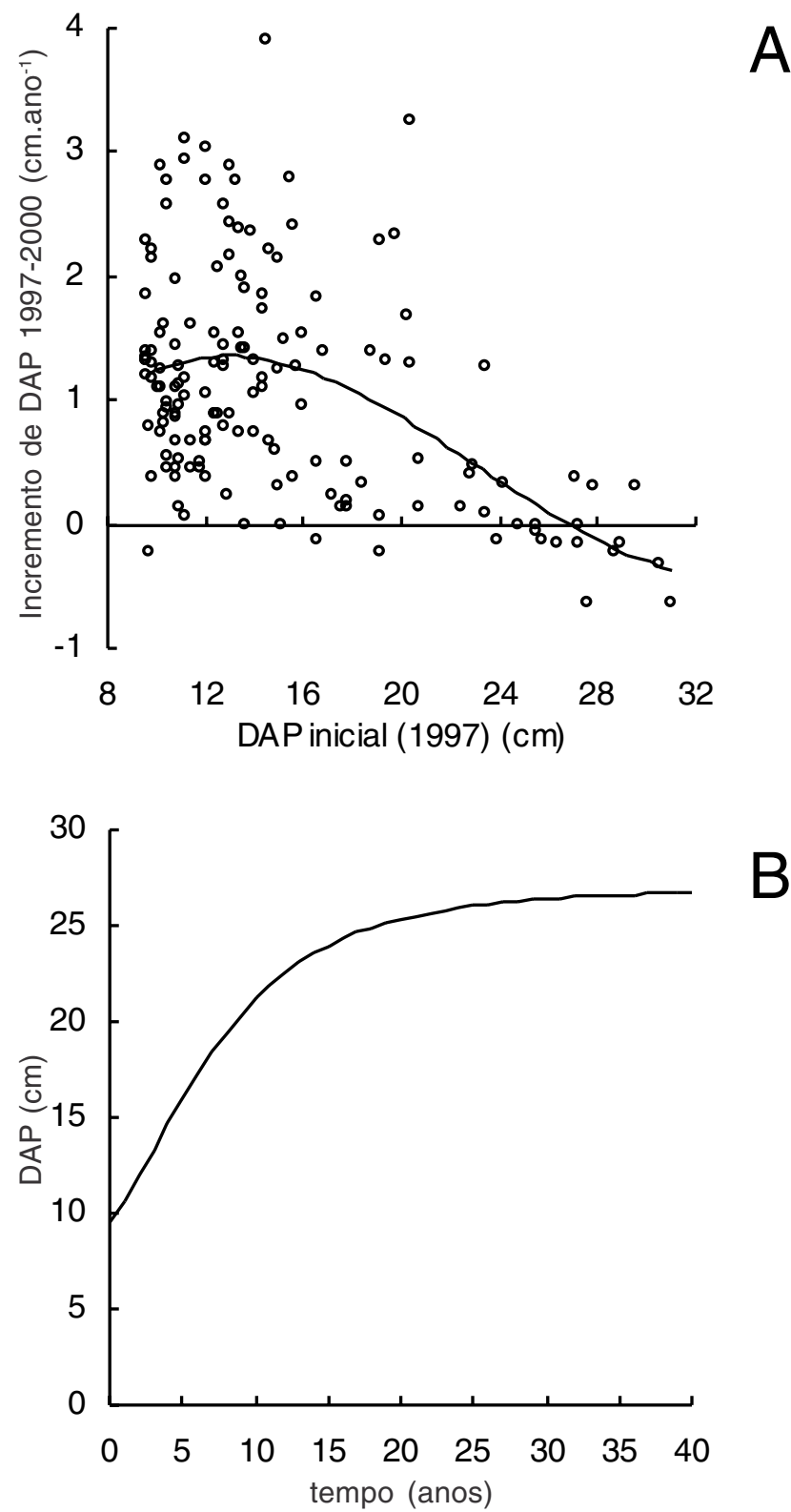

Figura 8. Incremento diamétrico de indivíduos de Archontophoenix cunninghamiana em função do DAP inicial. A função de melhor ajuste (linha contínua) tem a forma $y=0,0004 x^{3}-0,0297 x^{2}+0,5524 x-1,746 . R^{2}=0,2371$ (A). Modelo de aumento de DAP do indivíduo médio de $A$. cunninghamiana ao longo do tempo, a partir de DAP de $9,5 \mathrm{~cm}(\mathrm{~B})$. 
florestas tropicais. Por exemplo, as maiores taxas de crescimento populacional encontradas por Melo (2000), para árvores com DAP $\geq 9,9 \mathrm{~cm}$, em floresta na Ilha do Cardoso, SP, foram 5,96\%. $\mathrm{ano}^{-1}, 3,66 \%$. ano ${ }^{-1} \mathrm{e}$ $3,12 \%$. ano $^{-1}$ e as maiores taxas de recrutamento, $8,32 \%$.ano ${ }^{-1}, 4,46 \%$.ano ${ }^{-1}$ e 4,24\%.ano ${ }^{-1}$. Para Gomes (1998), as taxas de recrutamento mais altas registradas para árvores com DAP $>8 \mathrm{~cm}$, no Parque Estadual das Fontes do Ipiranga (São Paulo, SP) foram 12,63\%.ano ${ }^{-1}, 11,13 \%$. ano ${ }^{-1}$ e $10,66 \%$.ano ${ }^{-1}$. Em estudo anterior, analisando árvores com DAP $\geq 15,9 \mathrm{~cm}$, em uma área de 0,5 ha no interior da Reserva da CUASO, Dislich \& Pivello (no prelo) verificaram ser Archontophoenix cunninghamiana a espécie com maior recrutamento e crescimento populacional, dentre todas as 47 espécies analisadas.

As altas taxas de recrutamento e crescimento populacional apresentadas por Archontophoenix cunninghamiana são especialmente dignas de nota tendo-se em vista a já alta densidade da população na Reserva, conferindo-lhe inclusive o "status" de população mais abundante na Área 2 (DAP $\geq 9,5 \mathrm{~cm}$ ). O crescimento atual da população indica uma dominância ainda maior da espécie no futuro. Também os padrões de distribuição de freqüências de tamanhos suportam uma previsão neste sentido. Condit et al. (1998) demonstraram que apenas o formato da curva de distribuição de tamanhos não é capaz de prever a taxa de crescimento da população. Isto porque, para uma determinada taxa de crescimento populacional, um incremento diamétrico mais baixo, especialmente de indivíduos menores, e uma mortalidade mais alta também produzem distribuições mais inclinadas (com mais indivíduos menores e menos indivíduos maiores). Sendo assim, o simples fato da distribuição de DAP de A. cunninghamiana ser mais inclinada que a do resto da comunidade não garante, por si só, um crescimento maior. Se o incremento diamétrico da espécie fosse mais baixo e/ou a mortalidade fosse mais alta, a população de $A$. cunninghamiana poderia crescer menos que o resto da comunidade, levando a uma diminuição futura de sua abundância relativa. No entanto, A. cunninghamiana parece apresentar incremento diamétrico mais alto que o resto da comunidade na Reserva e mortalidade igual. Dislich \& Pivello (2002) obtiveram $2,10 \mathrm{~mm}$. ano ${ }^{-1}$ como a taxa média de incremento de DAP da comunidade (DAP $>15,9 \mathrm{~cm}$ ) em uma área no interior da Área 2. Para a mesma faixa de DAP, o presente estudo indica incremento diamétrico médio de 5,04 mm.ano ${ }^{-1}$ para A. cunninghamiana. Dislich \& Pivello (no prelo) obtiveram mortalidade de $0,87 \%$. ano $^{-1}$, enquanto o presente estudo indica mortali- dade de $0,88 \%$.ano ${ }^{-1}$ para $A$. cunninghamiana na mesma faixa de DAP. Estas condições levam a prever um crescimento maior da população de $A$. cunninghamiana em relação ao restante da comunidade, com conseqüente aumento de sua abundância relativa no futuro.

Embora pelo menos outras 29 espécies arbustivoarbóreas exóticas possam ser encontradas na Reserva (Rossi 1994), aparentemente apenas Archontophoenix cunninghamiana se tornou invasora. Este padrão, em que apenas uma pequena parte das espécies introduzidas em determinado local se torna invasora, concorda com o usualmente observado (Williamson 1996, Williamson \& Fitter 1996b).

Embora a Reserva Florestal da CUASO possua um histórico de perturbação antrópica, o que a tornaria mais suscetível a invasões biológicas, as características de Archontophoenix cunninghamiana aqui verificadas não permitem associar esta espécie a uma preferência por locais perturbados e/ou de maior luminosidade, ao contrário, a espécie virtualmente não ocorre nas regiões mais perturbadas da Reserva. O mapeamento dos indivíduos recrutados indica uma expansão da ocupação para o interior da Reserva, e observa-se o estabelecimento da espécie mesmo nas áreas mais sombreadas no interior da mata, o que não ocorre nas áreas mais abertas. Neste sentido, a ocorrência de perturbações pretéritas na Reserva não teria sido um fator preponderante para sua invasão por $A$. cunninghamiana. Por outro lado, a invasão por esta espécie parece ameaçar mais as áreas menos perturbadas da Reserva, justamente aquelas com maior valor em termos de conservação. Como conseqüência, recomenda-se especial atenção a esta espécie não apenas na própria Reserva Florestal da CUASO mas também em outros trechos de floresta, mesmo pouco perturbados, mas expostos a propágulos de $A$. cunninghamiana.

Em geral, fragmentos pequenos de hábitats naturais são mais suscetíveis a invasão biológica do que grandes áreas contínuas (Laurance \& Bierregaard 1997), provavelmente devido, entre outros fatores, à disponibilidade de propágulos de espécies invasoras provenientes do exterior do fragmento. É provável que este processo também esteja agindo no caso da Reserva da CUASO. As áreas ao seu redor, tanto as mais próximas, que fazem parte do campus da Universidade de São Paulo, quanto as um pouco mais distantes, formadas por bairros da cidade, apresentam grandes números de indivíduos adultos de Archontophoenix cunninghamiana, uma vez que esta espécie é amplamente cultivada como ornamental e utilizada na 
arborização urbana da cidade de São Paulo. As aves que se alimentam de seus frutos provavelmente promovem a dispersão das sementes mesmo a distâncias relativamente grandes. Desta forma, os indivíduos de A. cunninghamiana nos arredores da Reserva funcionam como fonte de propágulos para a invasão, além daqueles indivíduos adultos já presentes no interior da Reserva.

A análise do incremento diamétrico de Archontophoenix cunninghamiana indica que já ocorrem indivíduos na Reserva há pelo menos duas a três décadas, a idade estimada para os indivíduos maiores encontrados. No entanto, a grande maioria dos indivíduos com $\mathrm{DAP} \geq 9,5 \mathrm{~cm}$ são relativamente recentes, indicando uma intensificação do processo de estabelecimento da espécie na Reserva nos últimos dez anos. Esta mesma análise indica que a inexistência de indivíduos com DAP muito maior que $30 \mathrm{~cm}$ não se deve à falta de tempo para o incremento a diâmetros maiores, e sim ao diâmetro máximo alcançável, intrínseco à espécie.

Tendo em vista a alta taxa de crescimento populacional de Archontophoenix cunninghamiana, as altas densidades já alcançadas, a tendência de crescimento futuro da população e sua aparente capacidade de estabelecimento sob condições de baixa luminosidade, esta espécie apresenta uma tendência de aumento de impacto no interior da Reserva .

Desta forma, o presente estudo aponta para a necessidade de ações de manejo que visem o controle da população de Archontophoenix cunninghamiana na Reserva Florestal da CUASO, no intuito da conservação biológica no local. Para que sejam efetivas, estas ações devem também estender-se ao entorno da Reserva, buscando evitar a chegada de propágulos da espécie a partir de seu exterior.

Agradecimentos - Agradecemos aos alunos Alessandra Daniela Romagnoli, Renato Chimaso Yoshikawa, Ariel Renê Sampaio de Carvalho, Márcio Bernardino da Silva e Fábio Pinheiro, que colaboraram no trabalho de campo. Também registramos nosso agradecimento à FAPESP (processo n. 97/ 04495-0) e ao CNPq pelas bolsas de estudo concedidas respectivamente ao primeiro e ao segundo autores.

\section{Referências bibliográficas}

CERSÓSIMO, L.F. 1993. Variações espaciais e temporais no estabelecimento de plântulas em trecho de floresta secundária em São Paulo, SP. Dissertação de mestrado, Universidade de São Paulo, São Paulo.
CONDIT, R., SUKUMAR, R., HUBBELL, S.P. \& FOSTER, R.B. 1998. Predicting population trends from size distributions: a direct test in a tropical tree community. The American Naturalist 152:495-509.

CRONK, Q.C.B. \& FULLER, J.L. 1995. Plant invaders. Chapman $\&$ Hall, London.

DISLICH, R. \& PIVELLO, V.R. 2000. Tree dynamics of an urban tropical forest fragment (São Paulo, Brazil) during a five year interval. Boletim de Botânica da Universidade de São Paulo 20 (no prelo).

GOMES, E.P.C. 1998. Dinâmica do componente arbóreo de um trecho de mata em São Paulo, SP. Tese de Doutorado, Universidade de São Paulo, São Paulo.

GORRESIO-ROIZMAN, L. 1993. Fitossociologia e dinâmica do banco de sementes de populações arbóreas de floresta secundária em São Paulo, SP. Dissertação de mestrado, Universidade de São Paulo, São Paulo.

HASUI, E. \& HÖFLING, E. 1998. Preferência alimentar das aves frugívoras de um fragmento de floresta estacional semidecídua secundária, São Paulo, Brasil. Iheringia, Série Zoologia 84:43-64.

JOLY, A.B. 1950. Estudo fitogeográfico dos campos do Butantã. Boletim da Faculdade de Filosofia, Ciências e Letras da Universidade de São Paulo, Botânica 8:5-68.

KORNING, J. \& BALSLEV, H. 1994. Growth and mortality of trees in Amazonian tropical rain forest in Ecuador. Journal of Vegetation Science 5: 77-86.

LAURANCE, W.F. \& BIERREGAARD, JR., R.O. 1997. Tropical forest remnants. University of Chicago Press, Chicago.

LODGE, D.M. 1993. Biological invasions: lessons for ecology. Trends in Ecology and Evolution 8:133-137.

MEFFE, G.K. \& CARROLL, R. 1997. Principles of conservation biology. 2 ed. Sinawer Associates, Massachusetts.

MELO, M.M.R.F. 2000. Demografia de árvores em floresta pluvial tropical atlântica, Ilha do Cardoso, SP, Brasil. Tese de Doutorado. Universidade de São Paulo, São Paulo.

NOBLE, I.R. 1989. Attributes of invaders and the invading process: terrestrial and vascular plants. In Biological invasions: a global perspective (J.A. Drake, H.A. Mooney, F. di Castri, R.H. Groves, F.J. Kruger, M. Rejmanek \& M.H. Williamson, eds.). Wiley, Chichester, p.301-313.

PARKER, I.M., SIMBERLOFF, D., LONSDALE, W.M., GOODELL, K., WONHAM, M., KAREIVA, P.M., WILLIAMSON, M.H., VON HOLLE, B., MOYLE, P.B., BYERS, J.E. \& GOLDWASSER, L. 1999. Impact: toward a framework for understanding the ecological effects of invaders. Biological Invasions 1:3-19.

PETERS, H.A. 2001. Clidemia hirta invasion at the Pasoh Forest Reserve: an unexpected plant invasion in an undisturbed tropical forest. Biotropica 33:60-68.

PIRANI, J.R. \& CORTOPASSI-LAURINO, M. 1994. Flores e abelhas em São Paulo. 2 ed. Editora da Universidade de São Paulo, São Paulo. 
PIVELLO, V.R., CARVALHO, V.M.C., PECCININI, A.A.,LOPES, P.F. \& ROSSO, S. 1999a. Abundance and distribution of native and invasive alien grasses in a "cerrado" (Brazilian savanna) biological reserve. Biotropica 31:71-82.

PIVELLO, V.R., SHIDA, C.N. \& MEIRELLES, S.T. 1999b. Alien grasses in Brazilian savannas: a threat to the biodiversity. Biodiversity and Conservation 8:1281-1294.

PYSEK, P. 1995. On the terminology used in plant invasion studies. In Plant invasions: general aspects and special problems. (P. Pysek, K. Prach, M. Rejmanek \& M. Wade, eds.). SPB, Amsterdam, p.71-81.

PYSEK, P., PRACH, K., REJMANEK, M. \& WADE, M. 1995. Plant invasions: general aspects and special problems. SPB, Amsterdam.

REJMANEK, M. \& RICHARDSON, D.M. 1996. What attributes make some plant species more invasive? Ecology 77:1655-1661.

ROSSI, L. 1994. A flora arbóreo-arbustiva da mata da Reserva da Cidade Universitária "Armando de Salles Oliveira" (São Paulo, Brasil). Boletim do Instituto de Botânica de São Paulo 9:1-105.

ROY, J. 1990. In search of the characteristics of plant invaders. In Biological invasions in Europe and the Mediterranean Basin. (F. di Castri, A.J. Hansen \& M. Debussche, eds.). Kluwer Academic Publishers, Dordrecht, p.335-352.
RUESINK, J.L., PARKER, I.M., GROOM, M.J. \& KAREIVA, P.M. 1995. Reducing the risks of nonindigenous species introductions: guilty until proven innocent. BioScience 45:465-477.

SHOUTE, J.C. 1912. Über das Dickenwachstum der Palmen. Annales du Jardin Botanique de Buitenzorg (Sér. 2) 11:1-209.

TERBORGH, J., FLORES, C., MUELLER, P. \& DAVENPORT, L. 1997. Estimating the ages of successional stands of tropical trees from growth increments. Journal of Tropical Ecology 14:833-856.

VARANDA, E.M. 1977. Balanço hídrico de espécies da Mata do Butantã, São Paulo - SP. Dissertação de mestrado, Universidade de São Paulo, São Paulo.

VELOSO, H.P., RANGEL FILHO, A.L.R. \& LIMA, J.C.A. 1991. Classificação da vegetação brasileira, adaptada a um sistema universal. IBGE, Rio de Janeiro.

WATERHOUSE, J.T. \& QUINN, C.J. 1978. Growth patterns in the stem of the palm Archontophoenix cunninghamiana. Botanical Journal of the Linnean Society 77:73-93.

WILLIAMSON, M. 1996. Biological invasions. Chapman \& Hall, London.

WILLIAMSON, M. \& FITTER, A. 1996a. The characters of successful invaders. Biological Conservation 78:163-170.

WILLIAMSON, M. \& FITTER, A. 1996b. The varying success of invaders. Ecology 77:1661-1666. 